\title{
Bubble Strategy - A Practical Solution to Return to Regular Life in the Intertwined Era of Vaccine Rollouts and Virus Mutation
}

\author{
Ying Shen'; Da Huo'; Tong Yu'; Quanyi Wang ${ }^{1, *}$
}

\begin{abstract}
For a long time, vaccination and herd immunity were considered to be the magic solution for controlling coronavirus disease 2019 (COVID-19). However, the emergence of new variants altered people's expectations and prolonged the pandemic duration, especially the Omicron strain with substantially increased transmissibility and decreased vaccine efficacy. Therefore, we are in urgent need of a practical solution to resume regular life to some extent, while mitigating COVID-19 risks in the tight race between vaccine rollouts and virus variation. This commentary proposed that bubble strategy (or closed loop management), utilized in Tokyo 2020 Olympic Games and to be implemented in Beijing 2022 Winter Games, could serve as a novel technique of nonpharmaceutical interventions in coping with such a situation.
\end{abstract}

The second and final versions of the Playbooks for the Olympic and Paralympic Winter Games Beijing 2022 were jointly released on December 13, 2021 by the International Olympic Committee (IOC), International Paralympic Committee (IPC), and Beijing 2022 (1). With a "closed-loop management" strategy to be strictly implemented from before the Games to the end of the Paralympic Winter Games, the playbooks exhibited the resolution and confidence of IOC, IPC, and Beijing 2022 to deliver safe and successful Games. Based upon the dynamic of the COVID-Zero strategy towards COVID-19 currently in place in China and considering that local cases only occasionally emerged, the closed loop management implemented for Beijing 2022 is rather comprehensive and rigorous. Once an individual enters the loop, all his/her activities will be subject to closed loop management of the same standard covering accommodation, transport, catering, training, competitions, and arrival and departure, regardless of his/her identity as foreign nationals or domestic workers. A dedicated transport system will be established for the Games, through which the Games participants will be allowed to move between permitted destinations. Based on the current requirements outlined in the Playbooks, the closed loop is sophisticated and rigorous, and will undoubtedly serve as a model for bubble strategies in global mass gathering events in the context of heterogeneous COVID-19 risk potentials between countries.

In the intertwined era of vaccine rollouts and virus variations, bubble strategy has its special implications. For a long time, vaccination and herd immunity are considered to be the solution for COVID-19. Most studies have estimated the threshold for herd immunity as $60 \%-70 \%$ of total population vaccinated or infected (2). However, the emergence of virus variants, especially the newly emerged Omicron strain, altered people's expectation and may prolong the pandemic duration. The resurgence of COVID-19 in Manaus, Brazil was a recent example, where the Gamma strain severely hit the city that was already presumed as herd immunized by October 2020, infecting over $70 \%$ of the population (3). Similarly, a recent study in Massachusetts, the United State reported that almost $75 \%$ of infections (346 cases) in this study occurred in fully vaccinated individuals and the vaccination coverage among eligible Massachusetts residents had already reached $69 \%$ (4). In the context of measles, whose $R_{0}$ is as high as 12 to $18,95 \%$ vaccination coverage is sufficient to prevent local transmission (5). However, this might not be true for COVID-19 due to various reasons, although its $R_{0}$ is much lower at 1.9 to 6.5 (6-7). Constant virus mutations in COVID-19 hotspots along with vaccination hesitancy, uneven vaccine rollout status, breakthrough infections, and waning immunity make it possible that we will hardly achieve herd immunity in the foreseeable future. Therefore, we are in urgent need of a practical solution to resume regular life to some extent while mitigating COVID-19 risks in the tight race between vaccine 
rollouts and virus variation. The bubble strategy, or more accurately, the closed loop management to be implemented in the Beijing 2022 Games happens to be one.

There were already some successful bubble strategies in practice, such as the bubble to bubble management utilized in the Tokyo 2020 Games, which was held in a special circumstance where community transmission of COVID-19 was still rampant, and Japan was trapped in a state of emergency. On the day of the opening ceremony (July 23, 2021) alone, Tokyo reported 1,359 positive cases (8). Against such background, Figure 1 suggested that although there might have been some haphazard enforcement that may have caused leaking

Beijing 2022

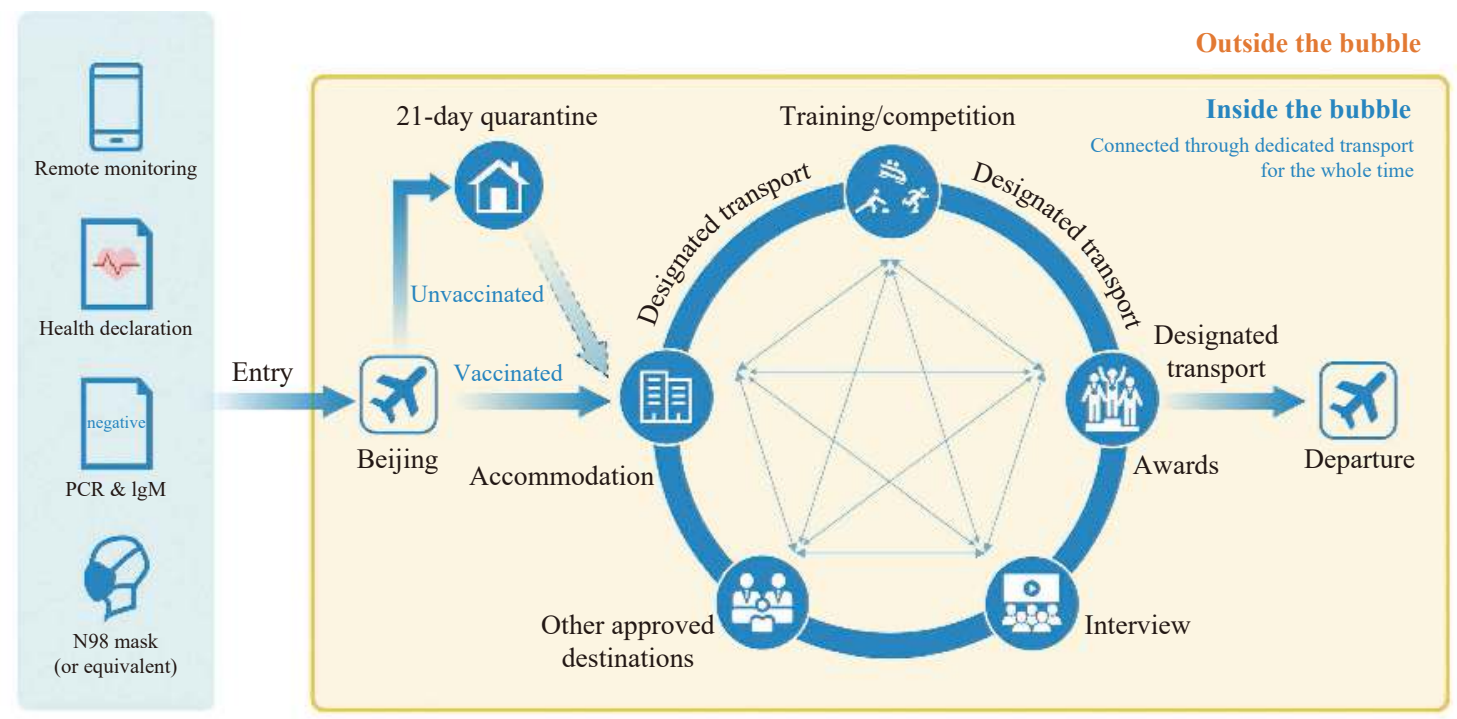

Tokyo 2020

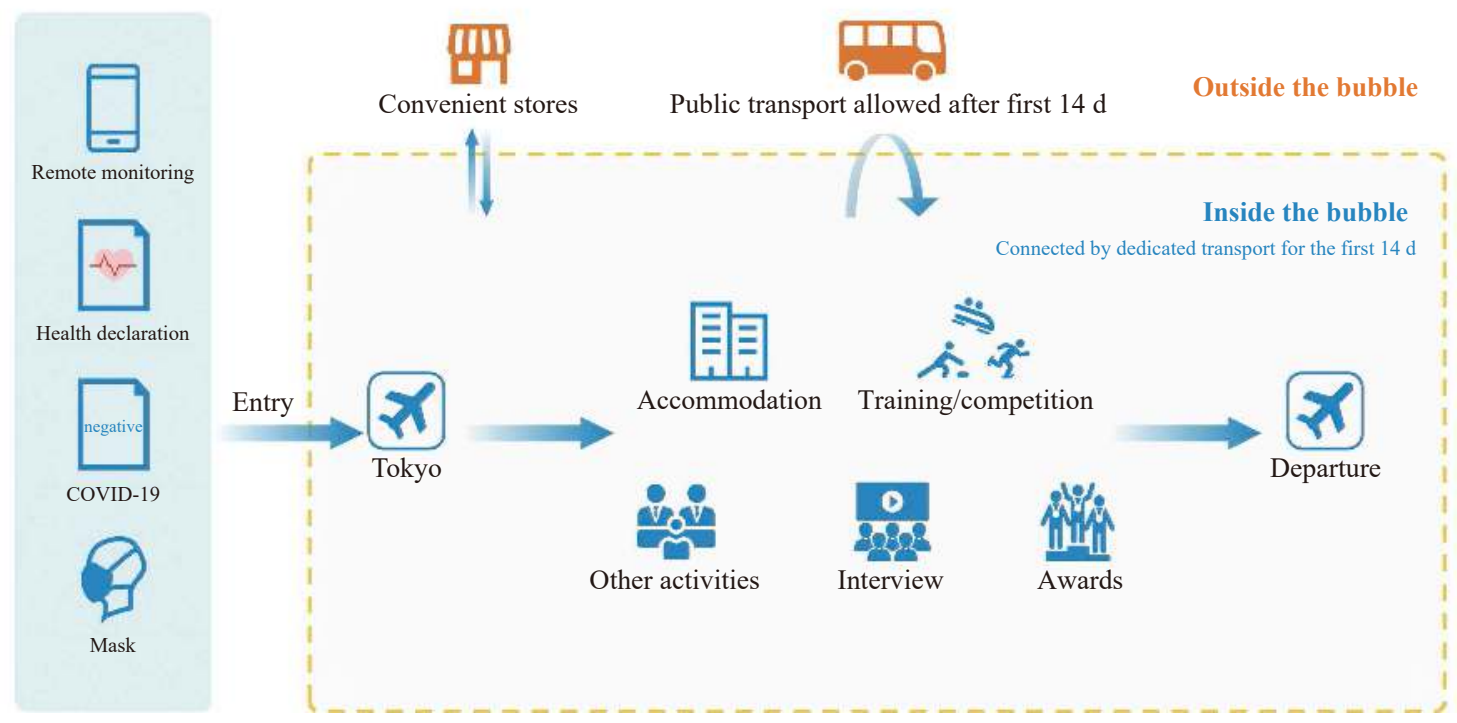

FIGURE 1. The figure depicted different bubble strategy used for Beijing 2022 Games and Tokyo 2020 Games.

Note: A rigorous closed-loop management system (or bubble strategy) will be applied for the Olympic and Paralympic Winter Games Beijing 2022 to all stakeholders from their pre-arrival days all the way to their departure from China. Within the bubble, stakeholders can only travel between pre-approved destinations via well-organized dedicated vehicles and interactions between those inside and outside of the bubble are strictly prohibited. The Tokyo 2020 Games, on the other hand, implemented a more compromised version of bubble strategy where stakeholders could temporarily leave the bubble for convenient stores, and were able to take public transport after 14 days in the bubble. 
out (9), the bubble strategy, along with other measures including vaccination and restricted social interactions, helped to effectively deliver a successful summer Olympic Games and offered an option to hold global major sporting events in the middle of a pandemic. Some might argue that compared to the bubble strategy for the Beijing 2022 Games, the Tokyo 2020 strategy was more penetrable as those in the bubble could easily get out and move around. However, the contexts of COVID-19 influenced the strategy employed in Beijing to that in Tokyo - Beijing is coping with sporadic and clusters of cases whereas Tokyo was in a state of emergency. Therefore, it is quite understandable that Beijing 2022 chooses to contain the virus with meticulous effort while Tokyo 2020 embraces a more compromised approach, which might be its best choice given its background.

From the two bubbles above, we can see that the bubble strategy is resilient in nature and each model of it could offer some potential to restore regular life while mitigating COVID-19 risks. Apart from the global mass gathering events such as sports competitions and business/academic conferences, the bubble strategy could also offer some opportunities for bilateral collaborations between international organizations or even between countries. For instance, to our knowledge, the most considerably successful bubble strategy implemented in record is the travel bubble (or travel corridors) between 2 or more countries at low risks for COVID-19. This is the case for New Zealand and Australia; through an exclusive partnership represented by travel bubbles, countries can re-establish connections and revive businesses by allowing people to travel freely with no requirements for upon-arrival quarantine. Furthermore, there are always some special occasions where low-risk individuals have to enter high risk regions. In this case, individuals of low-risk could intentionally establish a "protective bubble," through which they can restrict interactions with those outside of the bubble, minimizing the risk of infection. Furthermore, the bubble strategy could also be utilized in people's daily life in the world divided by severe acute respiratory syndrome coronavirus 2 (SARS-CoV-2). As pointed out by researchers, COVID-19 has substantially affected people's mental health and quality of life. By establishing social bubbles (or "social pods" or "quaranteams") between limited households, individuals are able to expand their social circles and carry out leisure activities, therefore restoring regular life to some extent and maintaining good mental health.

To conclude, bubble management is promising as a feasible strategy and a novel technique of nonpharmaceutical interventions when coping with emerging infectious diseases in the Olympic Games.

Conflicts of interest: No conflicts of interest.

Funding: National Key Research and Development Programme (2021YFF0306003); Scientific and Technological Innovation 2030 (2021ZD0114103).

doi: $10.46234 / \mathrm{ccdcw} 2022.014$

\# Corresponding author: Quanyi Wang, bjcdcxm@126.com.

${ }^{1}$ Beijing Center for Disease Prevention and Control, Beijing, China.

Submitted: January 13, 2022; Accepted: January 26, 2022

\section{REFERENCES}

1. International Olympic Committee, Playbook for athletes. IOC, 2021. https://stillmed.olympics.com/media/Documents/Olympic-Games/ Beijing-2022/Playbooks/The-Playbook-Athletes-and-Team-OfficialsDecember-2021.pdf?_ga=2.199131626.472829758.16432641101312700856.1622511275. [2021-10-22].

2. Aschwanden C. Five reasons why COVID herd immunity is probably impossible. Nature 2021;591(7851):520 - 2. http://dx.doi.org/10.1038/ d41586-021-00728-2.

3. Buss LF, Prete Jr CA, Abrahim CMM, Mendrone Jr A, Salomon T, De Almeida-Neto C, et al. Three-quarters attack rate of SARS-CoV-2 in the Brazilian Amazon during a largely unmitigated epidemic. Science 2021;371(6526):288 - 92. http://dx.doi.org/10.1126/science.abe9728.

4. Brown CM, Vostok J, Johnson H, Burns M, Gharpure R, Sami S, et al. Outbreak of SARS-CoV-2 infections, including COVID-19 vaccine breakthrough infections, associated with large public gatheringsbarnstable county, massachusetts, July 2021. MMWR Morb Mortal Wkly Rep 2021;70(31):1059 - 62. http://dx.doi.org/10.15585/mmwr. mm7031e2.

5. Guerra FM, Bolotin S, Lim G, Heffernan J, Deeks SL, Li Y, et al. The basic reproduction number $\left(\mathrm{R}_{0}\right)$ of measles: a systematic review. Lancet Infect Dis 2017;17(12):e420 - 8. http://dx.doi.org/10.1016/S1473-3099 (17)30307-9.

6. Hao XJ, Cheng SS, Wu DG, Wu TC, Lin XH, Wang CL. Reconstruction of the full transmission dynamics of COVID-19 in Wuhan. Nature 2020;584(7821):420 - 4. http://dx.doi.org/10.1038/ s41586-020-2554-8.

7. Ahammed T, Anjum A, Rahman MM, Haider N, Kock R, Uddin MJ. Estimation of novel coronavirus (COVID-19) reproduction number and case fatality rate: a systematic review and meta-analysis. Health Sci Rep 2021;4(2):e274. http://dx.doi.org/10.1002/hsr2.274.

8. Tokyo Metropolitan Government, Updates on COVID-19 in Tokyo. 2021. https://stopcovid19.metro.tokyo.lg.jp/en/monitoring. [2021-10$22]$.

9. Sparrow AK, Brosseau LM, Harrison RJ, Osterholm MT. Protecting olympic participants from covid-19-the urgent need for a riskmanagement approach. N Engl J Med 2021;385:e2. http://dx.doi.org/ 10.1056/NEJMp2108567. 